J. Dairy Sci. 95:2919-2934

http://dx.doi.org/10.3168/jds.2011-4239

(C) American Dairy Science Association ${ }^{\circledR}, 2012$.

\title{
A model of ruminal volatile fatty acid absorption kinetics and rumen epithelial blood flow in lactating Holstein cows
}

\author{
A. C. Storm, ${ }^{* 1}$ N. B. Kristensen, ${ }^{\star}$ and M. D. Hanigan $†$ \\ *Department of Animal Health and Bioscience, Faculty of Agricultural Sciences, Aarhus University, DK-8830 Tjele, Denmark \\ †Department of Dairy Science, Virginia Polytechnic Institute and State University, Blacksburg 24061
}

\begin{abstract}
Ruminal absorption of volatile fatty acids (VFA) is quantitatively the most important nutrient flux in cattle. Historically, VFA absorption models have been derived primarily from ruminal variables such as chemical composition of the fluid, volume, and $\mathrm{pH}$. Recently, a mechanistic model incorporated the control of VFA absorption from epithelial surface area of the reticulorumen. In the present study, we hypothesized that ruminal absorption of VFA was controlled through epithelial permeability to VFA and rumen epithelial capillary blood flow. The objective of the study was to construct a model of VFA exchange across the rumen wall that incorporates epithelial blood flow as a driving force for ruminal VFA removal. The bidirectional fluxes between the ruminal and epithelial pool of VFA were assumed mass action driven, given that passive diffusion of nonionized VFA is the dominant transmembrane VFA flux. Parameter estimates were derived by fitting the model to observed data. The model provided reliable unbiased estimates of ruminal VFA absorption and rumen epithelial blood flow. Blood flow was modeled using an equation that considered the effect of butyrate and dietary crude protein intake per kilogram of body weight. The rate constants related to the flux from ruminal fluid to epithelium were in the order isobutyrate $<$ acetate $<$ propionate $<$ butyrate $(0.32$ $\pm 0.02,0.72 \pm 0.2,0.91 \pm 0.06$, and $0.97 \pm 0.02 / \mathrm{h}$, respectively). The rate constants for fluxes of isobutyrate, acetate, propionate, and butyrate from the rumen epithelium to the ruminal fluid, relative to the pool size of the epithelium, were $4.78,10.6,13.4$, and $14.3 / \mathrm{h}$, respectively. Ruminal concentrations of acetate, propionate, butyrate, and isobutyrate were predicted with root mean square prediction errors as percentage of the observed means (RMSPE) of 5.86, 5.75, 11.3, and 4.12, respectively. The epithelial blood flow was predicted with $26.3 \%$ RMSPE. Sensitivity analyses indicated
\end{abstract}

Received February 5, 2011.

Accepted January 22, 2012.

${ }^{1}$ Corresponding author: Adam.Storm@agrsci.dk that when ruminal butyrate concentration increased from 4.0 to $37.4 \mathrm{mmol} / \mathrm{L}$, blood flow of the epithelium increased $47 \%$ and the ruminal disappearance rate of propionate increased $11 \%$. The concentration gradient of propionate between ruminal fluid and epithelium was no more than 3:1 and increased with increasing blood flow. In conclusion, a dynamic model based on rumen epithelial blood flow and bidirectional fluxes of VFA between ruminal fluid and epithelium gave unbiased predictions with low residual error of ruminal VFA absorption under washed rumen conditions. The model indicates that the effect of varying epithelial blood flow on the control of ruminal VFA absorption is related to the concentration gradient of individual VFA between ruminal fluid and epithelial blood. Epithelial blood flow may be an important determinant of ruminal absorption of VFA, a result that has not been evaluated on independent data.

Key words: dairy cow, volatile fatty acid model, epithelium blood flow, ruminal volatile fatty acid kinetics

\section{INTRODUCTION}

Volatile fatty acids are quantitatively the most important end products of ruminal fermentation and provide the cow with the majority of energetic precursors for metabolic processes. On a net basis, dairy cows absorb approximately $100 \mathrm{~mol}$ of VFA per day (Storm and Kristensen, 2010, 2011). When measured in beef cattle, 85 to $100 \%$ of ruminal VFA is absorbed across stomach tissue (Reynolds and Huntington, 1988). Modeling utilization of specific nutrients for animal gain (meat and adipose), reproduction (placenta and fetus), and milk relies on accurate prediction of the available nutrients. Predictions of ruminal production and absorption of VFA by existing mechanistic models have been shown not to fit today's high-yielding dairy cows (Bannink et al., 1997b), and the inaccuracies are presumably caused by poor stoichiometric predictions of VFA production (Bannink et al., 1997a). The set of stoichiometric equations originally used were derived from data from various ruminant species, but few of these data were from lactating dairy cows (Murphy et al., 1982). Bannink et 
al. (2006) derived a new set of stoichiometric equations from data on lactating Friesian cows in an attempt to improve the prediction of ruminal molar production of VFA and subsequently modeled the effect of ruminal cell proliferation and metabolic activity in relation to properties of the ruminal fluid (Bannink et al., 2008). In a recent study, 6 ruminal VFA stoichiometry models were evaluated against independent data and indicated that ruminal production of VFA is not a simple linear relationship between substrate and $\mathrm{pH}$ (Morvay et al., 2011). Morvay et al. (2011) speculated that one possible bias in the prediction of ruminal molar proportion might relate to a bias in the representation of ruminal absorption in these models.

The absorption mechanism for VFA is still not fully elucidated and several theories exist. Passive diffusion of nonionized VFA across ruminal epithelium is the most common theory but protein-mediated absorption of ionized VFA has been identified as a possible alternative to passive diffusion (for reviews, see Bergman, 1990; Bugaut, 1987; Dijkstra, 1994; Aschenbach et al., 2011). A poor representation of the absorption will bias predictions of ruminal VFA pools, consequently affecting estimates of ruminal $\mathrm{pH}$. Both ruminal $\mathrm{pH}$ and VFA pool size are important for modeling fermentation. Historically, VFA absorption models have been derived primarily from ruminal variables such as chemical composition of the fluid, volume, and $\mathrm{pH}$ (Baldwin et al., 1987; Danfær, 1990; Dijkstra, 1993). Bannink et al. (2008) was the first to incorporate a specific effect of the cow in the control of VFA absorption by using epithelial surface area of the reticulorumen in the model. In a recent study, we observed that manipulation of the ruminal epithelial blood flow affects ruminal disappearance and net portal appearance of propionate (Storm et al., 2011). Hence, the absorption of VFA is affected both by epithelial permeability to VFA and by blood flow through the active absorptive epithelium of the rumen. Previous studies have shown that ruminal butyrate concentration is an important determinant of epithelial blood flow rate (Dobson et al., 1971) and ruminal vein blood flow rate (Rémond et al., 1993), which was in agreement with Storm et al. (2011), who also observed that dietary CP intake (CPI) influenced epithelial blood flow rate. Contradicting the effect of blood flow on ruminal absorption of VFA is the study by Kristensen and Harmon (2004a), which found no effect on ruminal disappearance of propionate in steers when ruminal butyrate concentrations were increased from 4 to $36 \mathrm{mmol} / \mathrm{L}$. The contradicting result of Kristensen and Harmon (2004a) might relate to steers being less metabolically active compared with lactating dairy cows. Storm et al. (2011) suggested that models of VFA absorption could be improved by describing rumen fluid to rumen epithelial VFA exchange using bidirectional VFA fluxes and rumen epithelial blood flow as a determinant of VFA exchange across the rumen wall.

The hypothesis of the present study was that ruminal absorption of VFA was controlled by epithelial permeability to VFA and rumen epithelial capillary blood flow. The objective of the present study was to construct a model of VFA exchange across the rumen wall that incorporates epithelial blood flow as a driving force for ruminal VFA removal.

\section{MATERIALS AND METHODS}

Data used in the present study were obtained from a washed rumen experiment with multicatheterized lactating Holstein cows (Storm et al., 2011). Cows were fed a low or high CP diet (high CP 17.1\%, low CP $12.9 \%$ ) as described previously (Kristensen et al., 2010). The experimental treatment structure was a split-plot design with CP level as the whole-plot factor and buffer treatment as sub-plot factor. For each buffer sequence (wash buffer followed by treatment buffer), 5 sets of arterial blood, hepatic-portal vein blood, right ruminal vein blood, and ruminal buffer samples were collected simultaneously. Treatment buffers contained 22.0, 2.0, and $1.2 \mathrm{mmol} / \mathrm{L}$ of propionate, isobutyrate, and valerate, respectively. Treatment buffers differed in acetate, butyrate, and ammonia concentrations (control: 55.0, 4.0, and $2.5 \mathrm{mmol} / \mathrm{L}$; high butyrate: $23.0,36.0$, and 2.5 $\mathrm{mmol} / \mathrm{L}$; high ammonia 55.0, 4.0, and $22.5 \mathrm{mmol} / \mathrm{L}$, respectively); a full description of the buffer compositions is presented in Storm et al. (2011). Because the control and high ammonia treatments did not differ in their effects on epithelial blood flow and ruminal disappearance of propionate, data from these treatments were combined into one control treatment $(\mathrm{n}=16)$ for the current presentation of results. The data set used to fit the model consisted of 24 sets of epithelial blood flows at time points 9,20 , and 30 min after ruminal introduction of the treatment buffer together with ruminal concentrations of acetate, propionate, butyrate, and isobutyrate at time points $3,9,20$, and $30 \mathrm{~min}$. Mean arterial blood VFA concentrations across time points $3,9,20$, and 30 min and initial treatment buffer concentrations of VFA together with ruminal fractional passage rate, saliva influx, residual water in the rumen upon buffer entry, CPI, and BW from the washed rumen study described in Storm et al. (2011) were used as input data.

\section{Model Derivation}

The model describes VFA absorption from the rumen of a lactating dairy cow using ruminal epithelium 
blood flow to model the interaction between ruminal VFA concentrations and VFA kinetics. The model is based on a set of dynamic differential equations coded in Advanced Continuous Simulation Language Extreme (acslX; Aegis Technologies Group Int., Huntsville, $\mathrm{AL})$. The model includes a ruminal $(\boldsymbol{R} \boldsymbol{u})$ and an epithelial ( $\boldsymbol{E p})$ unit (including extracellular and intracellular space). These physiological units are subdivided into pools of acetate $(\boldsymbol{A c})$, propionate $(\boldsymbol{P r})$, butyrate $(\boldsymbol{B u})$, and isobutyrate $(\boldsymbol{I} \boldsymbol{b} \boldsymbol{u})$, giving 8 primary state variables. An auxiliary variable of ruminal fluid $(\boldsymbol{A} \boldsymbol{q})$ is also included in the model. A flow diagram of the model is provided in Figure 1. The following expressions and units were used: mass $(\boldsymbol{Q}, \mathrm{mmol})$, volume $(\boldsymbol{V}$, $\mathrm{L}$ ), concentration $(\boldsymbol{C}, \mathrm{mmol} / \mathrm{L})$, flux $(\boldsymbol{F}, \mathrm{mmol} / \mathrm{h})$, epithelial blood flow $(\boldsymbol{B F}, \mathrm{L} / \mathrm{h})$, fluid flow $(\boldsymbol{F} \boldsymbol{L}, \mathrm{L} / \mathrm{h})$, rate constant $\left[\boldsymbol{k} ; k_{B F C P I},(\mathrm{~L} \times \mathrm{d}) / \mathrm{h}\right.$, and $k_{B F B u}, \mathrm{~L}^{3} /\left(\mathrm{mmol}^{2}\right.$ $\times \mathrm{h}$ ); all other $k$ s have the unit $/ \mathrm{h}]$, and time $(\boldsymbol{t}, \mathrm{h})$. Abbreviations follow the system of $H_{i j}$ unless otherwise stated, where $H$ describes the variable type $(Q, V, C$, $F, B F, F L, k)$ and $i j$ the substrates and pools involved (i.e., $F_{E p A c, R u A c}$ is the flux of acetate to the epithelial acetate pool from the ruminal acetate pool). State variables, flow, and fluxes are explained in Table 1. Arterial blood $(\boldsymbol{A} \boldsymbol{b})$, ruminal vein blood $(\boldsymbol{R} \boldsymbol{V} \boldsymbol{b})$, saliva $(\boldsymbol{S a l})$, passage (Pas) out of the rumen to the omasum, and ruminal buffer $(\boldsymbol{B} \boldsymbol{u} \boldsymbol{f})$ are also parameters in the model. In the following description of equations used in the model, VFA is used in the abbreviations as a substitute for the different VFA (Ac, Pr, Bu, and Ibu). The differential equations describing changes in the pool of $V_{R u A q}, Q_{R u A c}, Q_{R u P r}, Q_{R u B u}, Q_{R u I b u}, Q_{E p A c}, Q_{E p P r}, Q_{E p B u}$, and $Q_{E p I b u}$ are represented in Eq. [1] to Eq. [3]:

$$
\begin{aligned}
& \frac{\mathrm{d} V_{R u A q}}{\mathrm{~d} t}=F L_{R u A q, S a l A q}-F L_{P a s A q, R u A q}, \\
& \frac{\mathrm{d} Q_{R u V F A}}{\mathrm{~d} t}=F_{R u V F A, S a l V F A}+F_{R u V F A, E p V F A} \\
& \text { - } F_{\text {Pas VFA,RuVFA }}-F_{E p V F A, R u V F A} \text {, } \\
& \frac{\mathrm{d} Q_{E p V F A}}{\mathrm{~d} t}=F_{E p V F A, R u V F A}+F_{E p V F A, A b V F A} \\
& -F_{R u V F A, E p V F A}-F_{R V b V F A, E p V F A} .
\end{aligned}
$$

Rumen epithelial volume $\left(\boldsymbol{V}_{\boldsymbol{E} p}\right)$ was considered a constant $2.123 \mathrm{~L}$, corresponding to the epithelial weight of ventral rumen, atrium, and reticulum in a sheep extrapolated to a cow of $565 \mathrm{~kg}$ (Barnes et al., 1983). The epithelial pool was the combined water space of intracellular and extracellular space and was assumed to be in equilibrium with respect to VFA. The concentrations of VFA in the rumen (Eq. [4]) and epithelium (Eq. [5]) were calculated as

$$
\begin{gathered}
C_{R u V F A}=\frac{Q_{R u V F A}}{V_{R u A q}}, \\
C_{E p V F A}=\frac{Q_{E p V F A}}{V_{E p}} .
\end{gathered}
$$

The salivary flow $\left(\boldsymbol{F} \boldsymbol{L}_{\boldsymbol{R u A q}, \text { SalAq }}\right)$ and the salivary flux of VFA (Eq. [6]) into the rumen were assumed constant. Salivary VFA concentrations were assumed equal to initial arterial plasma VFA concentrations. The passage of ruminal fluid (Eq. [7]) and VFA (Eq. [8]) out of the rumen to the omasum and the VFA fluxes entering (Eq. [9]) and leaving (Eq. [10]) the epithelium from the rumen were assumed driven by mass action. Fractional passage rate of VFA from the rumen to the omasum $\left(\boldsymbol{k}_{\text {pas } V F A, R u V F A}\right)$ was considered equal to the liquid fractional passage rate $\left(\boldsymbol{k}_{\text {pasAq, } R u A q}\right)$.

$$
\begin{gathered}
F_{R u V F A, S a l V F A}=F L_{R u A q, S a l A q} \times C_{A b V F A}, \\
F L_{P a s A q, R u A q}=k_{P a s A q, R u A q} \times V_{R u A q}, \\
F_{P a s V F A, R u V F A}=k_{P a s V F A, R u V F A} \times Q_{R u V F A}, \\
F_{E p V F A, R u V F A}=k_{E p V F A, R u V F A} \times Q_{R u V F A}, \\
F_{R u V F A, E p V F A}=k_{R u V F A, E p V F A} \times Q_{E p V F A} .
\end{gathered}
$$

The arterial input (Eq. [11]) and the venous output (Eq. [12]) of VFA from the epithelium were described as the product of epithelial blood flow and the arterial and epithelial concentrations of VFA, respectively:

$$
\begin{gathered}
F_{E p V F A, A b V F A}=B F \times C_{A b V F A}, \\
F_{R V b V F A, E p V F A}=B F \times C_{E p V F A} .
\end{gathered}
$$

Reticulorumen epithelial blood flow in the active absorptive epithelium was affected by ruminal butyrate concentration and CPI in the washed rumen study (Storm et al., 2011). The equation for calculation of epithelial blood flow (Eq. [13]) therefore considers the effects of CPI per kilogram of BW and ruminal butyrate concentration. The blood flow was set up with a basal blood flow controlled by CPI and BW and a variable blood flow affected by the quadratic ruminal butyrate 

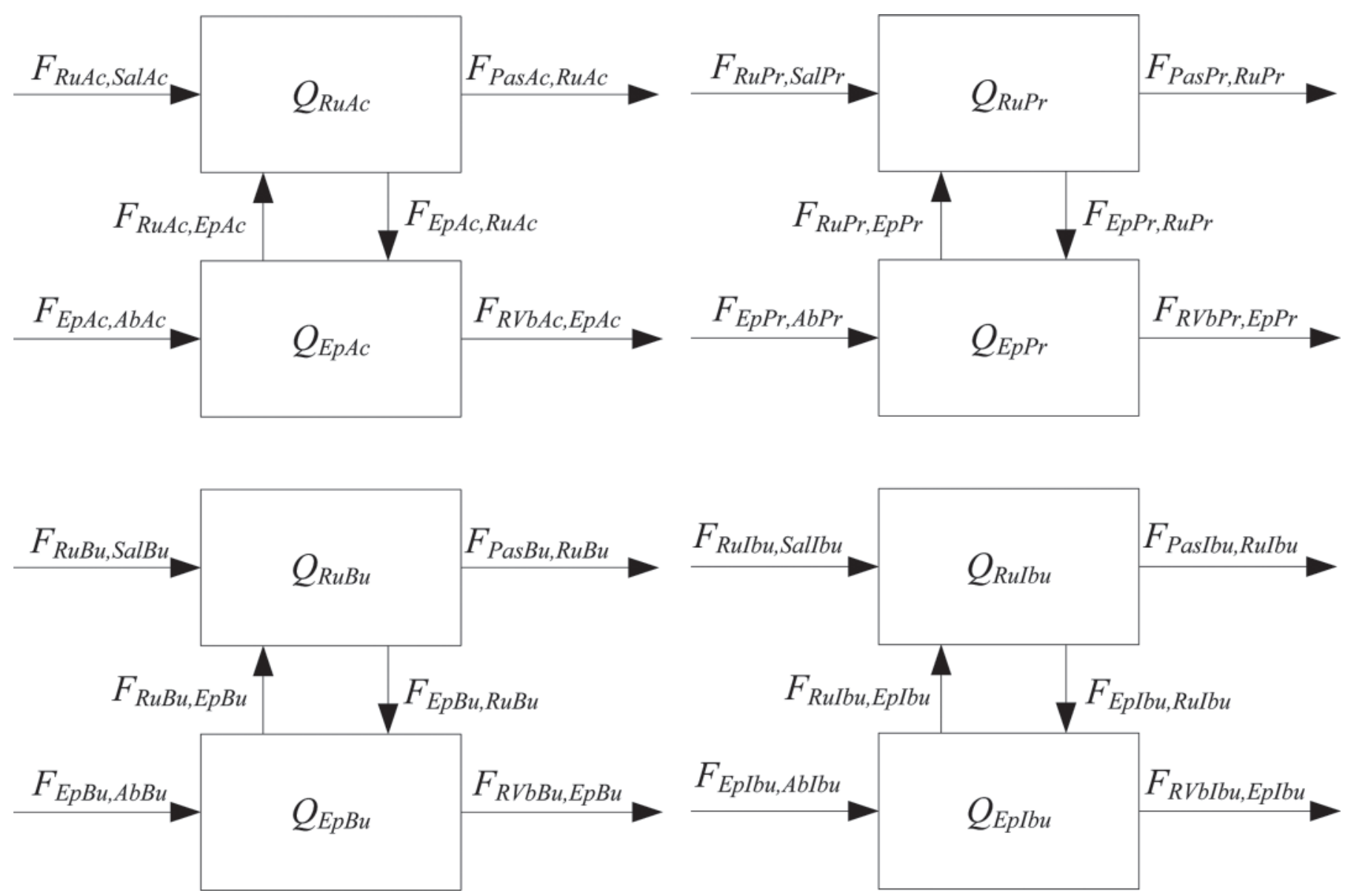

Figure 1. Flow diagram of the ruminal absorption model. Boxes represent pools and arrows represent fluxes between pools; abbreviations are explained in Table 1.

concentration. Body weight was used as a parameter to account for a possible variation in epithelial blood flow between cows of different size:

$$
B F=k_{B F C P I} \times \frac{C P I \times 1,000}{B W}+k_{B F B u} \times C_{R u B u}^{2} .
$$

The ruminal pool of fluid $\left(\boldsymbol{V}_{\boldsymbol{R} u A q}\right)$, ruminal pools of different VFA $\left(\boldsymbol{Q}_{\boldsymbol{R} u \boldsymbol{V} \boldsymbol{F A}}\right)$, and epithelial pool of different VFA $\left(\boldsymbol{Q}_{\boldsymbol{E} \boldsymbol{V} \boldsymbol{V} \boldsymbol{F A}}\right)$ were derived by numerical integration of the respective differential equations starting from the initial $(\boldsymbol{i})$ pool sizes (Eq. [14], [15], and [16], respectively). Initial ruminal pools of VFA $\left(i Q_{R u V F A}\right.$ : Eq. [17]) were calculated using the initial buffer concentrations $\left(\boldsymbol{C}_{\text {BufVFA }}\right)$ and the initial buffer volume $\left(\boldsymbol{i} \boldsymbol{V}_{\boldsymbol{B u f}}: 30\right.$ L). The initial ruminal volume of liquid ( $\boldsymbol{i} \boldsymbol{V}_{\boldsymbol{R} u \boldsymbol{A}}$ : Eq. [18]) was calculated by adding the $\boldsymbol{i} \boldsymbol{V}_{\boldsymbol{B u f}}$ to the residual water in the rumen upon buffer entry $\left(\boldsymbol{i} \boldsymbol{V}_{\boldsymbol{R} u \boldsymbol{H} 2 \boldsymbol{O}}\right)$. Initial epithelium pools of VFA (Eq. [19]) were calculated assuming the tissue to equilibrate with arterial blood, because the cows were subjected to a 20-min period without VFA in the rumen before introduction of VFA buffers. A fourth-order, Runga-Kutta fixed-step integration algorithm was used with a step size of $0.01 \mathrm{~h}$ :

$$
\begin{gathered}
V_{R u A q}=\int_{0}^{t} \mathrm{~d} V_{R u A q}+i V_{R u A q}, \\
Q_{R u V F A}=\int_{0}^{t} \mathrm{~d} Q_{R u V F A}+i Q_{R u V F A}, \\
Q_{E p V F A}=\int_{0}^{t} \mathrm{~d} Q_{E p V F A}+i Q_{E p V F A}, \\
i Q_{R u V F A}=C_{B u f V F A} \times i V_{B u f}, \\
i V_{R u A q}=i V_{B u f}+i V_{R u H 2 O}, \\
i Q_{E p V F A}=C_{A b V F A} \times V_{E p} .
\end{gathered}
$$


Table 1. Abbreviations, description, and unit of model state variables, flow, and fluxes

\begin{tabular}{|c|c|c|c|}
\hline Item & Description & Eq. & Unit \\
\hline$V_{R u, A q}$ & Ruminal fluid volume & [1] & $\mathrm{L}$ \\
\hline$Q_{R u, A c}$ & Ruminal quantum of acetate & {$[2]$} & mmol \\
\hline$Q_{R u, P r}$ & Ruminal quantum of propionate & {$[2]$} & mmol \\
\hline$Q_{R u, B u}$ & Ruminal quantum of butyrate & {$[2]$} & mmol \\
\hline$Q_{R u, I b u}$ & Ruminal quantum of isobutyrate & {$[2]$} & mmol \\
\hline$Q_{E p, A c}$ & Epithelial quantum of acetate & {$[3]$} & mmol \\
\hline$Q_{E p, P r}$ & Epithelial quantum of propionate & {$[3]$} & $\mathrm{mmol}$ \\
\hline$Q_{E p, B u}$ & Epithelial quantum of butyrate & [3] & mmol \\
\hline$Q_{E p, I b u}$ & Epithelial quantum of isobutyrate & {$[3]$} & mmol \\
\hline$F L_{R u A q, S a l A q}$ & Flow of saliva into the rumen & - & $\mathrm{L} / \mathrm{h}$ \\
\hline$F_{\text {RuAc,SalAc }}$ & Flux of acetate to the rumen from saliva & {$[6]$} & $\mathrm{mmol} / \mathrm{h}$ \\
\hline$F_{\text {RuPr,SalPr }}$ & Flux of propionate to the rumen from saliva & {$[6]$} & $\mathrm{mmol} / \mathrm{h}$ \\
\hline$F_{R u B u, S a l B u}$ & Flux of butyrate to the rumen from saliva & {$[6]$} & $\mathrm{mmol} / \mathrm{h}$ \\
\hline$F_{\text {RuIbu,Sallbu }}$ & Flux of isobutyrate to the rumen from saliva & {$[6]$} & $\mathrm{mmol} / \mathrm{h}$ \\
\hline$F L_{P a s A q, R u A q}$ & Flow of ruminal fluid to the omasum from rumen as passage & {$[7]$} & $\mathrm{L} / \mathrm{h}$ \\
\hline$F_{P a s A c, R u A c}$ & Flux of acetate to the omasum from rumen as passage & {$[8]$} & $\mathrm{mmol} / \mathrm{h}$ \\
\hline$F_{P a s P r, R u P r}$ & Flux of propionate to the omasum from rumen as passage & [8] & $\mathrm{mmol} / \mathrm{h}$ \\
\hline$F_{P a s B u, R u B u}$ & Flux of butyrate to the omasum from rumen as passage & [8] & $\mathrm{mmol} / \mathrm{h}$ \\
\hline$F_{\text {PasIbu,RuIbu }}$ & Flux of isobutyrate to the omasum from rumen as passage & [8] & $\mathrm{mmol} / \mathrm{h}$ \\
\hline$F_{E p A c, R u A c}$ & Flux of acetate to the epithelium from rumen & {$[9]$} & $\mathrm{mmol} / \mathrm{h}$ \\
\hline$F_{E p P r, R u P r}$ & Flux of propionate to the epithelium from rumen & {$[9]$} & $\mathrm{mmol} / \mathrm{h}$ \\
\hline$F_{E p B u, R u B u}$ & Flux of butyrate to the epithelium from rumen & [9] & $\mathrm{mmol} / \mathrm{h}$ \\
\hline$F_{E p I b u, R u I b u}$ & Flux of isobutyrate to the epithelium from rumen & {$[9]$} & $\mathrm{mmol} / \mathrm{h}$ \\
\hline$F_{R u A c, E p A c}$ & Flux of acetate to the rumen from the epithelium & {$[10]$} & $\mathrm{mmol} / \mathrm{h}$ \\
\hline$F_{R u P r, E p P r}$ & Flux of propionate to the rumen from the epithelium & {$[10]$} & $\mathrm{mmol} / \mathrm{h}$ \\
\hline$F_{R u B u, E p B u}$ & Flux of butyrate to the rumen from the epithelium & {$[10]$} & $\mathrm{mmol} / \mathrm{h}$ \\
\hline$F_{\text {RuIbu, EpIbu }}$ & Flux of isobutyrate to the rumen from the epithelium & [10] & $\mathrm{mmol} / \mathrm{h}$ \\
\hline$F_{E p A c, A b A c}$ & Flux of acetate to the epithelium from arterial blood & {$[11]$} & $\mathrm{mmol} / \mathrm{h}$ \\
\hline$F_{E p P r, A b P r}$ & Flux of propionate to the epithelium from arterial blood & {$[11]$} & $\mathrm{mmol} / \mathrm{h}$ \\
\hline$F_{E p B u, A b B u}$ & Flux of butyrate to the epithelium from arterial blood & {$[11]$} & $\mathrm{mmol} / \mathrm{h}$ \\
\hline$F_{E p I b u, A b I b u}$ & Flux of isobutyrate to the epithelium from arterial blood & [11] & $\mathrm{mmol} / \mathrm{h}$ \\
\hline$F_{R V b A c E p A c}$ & Flux of acetate to the ruminal vein blood from the epithelium & {$[12]$} & $\mathrm{mmol} / \mathrm{h}$ \\
\hline$F_{R V b P r, E p P r}$ & Flux of propionate to the ruminal vein blood from the epithelium & {$[12]$} & $\mathrm{mmol} / \mathrm{h}$ \\
\hline$F_{R V b B u, E p B u}$ & Flux of butyrate to the ruminal vein blood from the epithelium & {$[12]$} & $\mathrm{mmol} / \mathrm{h}$ \\
\hline$F_{R V b I b u, E p I b u}$ & Flux of isobutyrate to the ruminal vein blood from the epithelium & {$[12]$} & $\mathrm{mmol} / \mathrm{h}$ \\
\hline
\end{tabular}

\section{Parameter Estimation}

The model rate constants were fitted to observed data using the Nelder-Mead optimization algorithm resident in acslX while maximizing the log-likelihood function. Initial parameter values were calculated from log-transformations of the observed data.

Observed data used for the optimizer were the longitudinal data of ruminal acetate, propionate, butyrate, and isobutyrate concentrations, as well as rumen epithelial blood flow. Input data was set to the values present in Table 2 and the fixed epithelium volume. The volume of rumen fluid was affected by passage to distal compartments and saliva secretion. Ruminal passage, saliva secretion, and residual ruminal water were estimated for each combination of cow and buffer, as described previously (Storm et al., 2011) and used as inputs to the model. The bidirectional fluxes of VFA across the rumen wall (Eq. [9], [10], respectively) were assumed restricted by the epithelial permeability, which was assumed the same for both transport directions. Therefore, only one rate constant was to be estimated because the other could be calculated using Eq. [20] and 2 assumptions: (1) when epithelial blood flow is equal to $0 \mathrm{~L} / \mathrm{h}$, the efflux was equal to the influx of VFA; and (2) when epithelial blood flow is equal to 0 $\mathrm{L} / \mathrm{h}$, the ruminal VFA concentration will be in equilibrium with the epithelium:

$$
k_{R u V F A, E p V F A}=\frac{i Q_{R u V F A}}{i C_{R u V F A} \times V_{E p}} \times k_{E p V F A, R u V F A} .
$$

From the above-mentioned inputs, the model predicted rumen epithelial blood flow. The model also predicted the bidirectional fluxes between the rumen and epithelial VFA pools together with the VFA concentration in these pools.

\section{Calculations and Statistical Analysis}

Sensitivity analysis was performed on the final model with variation of model inputs of ruminal acetate and butyrate to estimate the effect of increasing ruminal 
Table 2. Inputs to the model divided in control and high butyrate represented as means $( \pm \mathrm{SD})$ of the observed data of the washed rumen study (Storm et al., 2011)

\begin{tabular}{|c|c|c|c|}
\hline Item & Description & $\begin{array}{l}\text { Control } \\
(\mathrm{n}=16)\end{array}$ & $\begin{array}{l}\text { High butyrate } \\
\quad(\mathrm{n}=8)\end{array}$ \\
\hline$C_{A b A c}$ & Arterial acetate, $\mathrm{mmol} / \mathrm{L}$ & $0.74 \pm 0.17$ & $0.55 \pm 0.14$ \\
\hline$C_{A b P r}$ & Arterial propionate, $\mathrm{mmol} / \mathrm{L}$ & $0.022 \pm 0.016$ & $0.029 \pm 0.014$ \\
\hline$C_{A b B u}$ & Arterial butyrate, $\mathrm{mmol} / \mathrm{L}$ & $0.0041 \pm 0.0025$ & $0.037 \pm 0.011$ \\
\hline$C_{A b l b u}$ & Arterial isobutyrate, $\mathrm{mmol} / \mathrm{L}$ & $0.0020 \pm 0.0010$ & $0.0024 \pm 0.0012$ \\
\hline$C_{B u f A c}$ & Initial buffer acetate, $\mathrm{mmol} / \mathrm{L}$ & $54.9 \pm 1.5$ & $22.9 \pm 0.9$ \\
\hline$C_{B u f P r}$ & Initial buffer propionate, $\mathrm{mmol} / \mathrm{L}$ & $22.2 \pm 0.4$ & $22.4 \pm 0.6$ \\
\hline$C_{B u f B u}$ & Initial buffer butyrate, $\mathrm{mmol} / \mathrm{L}$ & $4.01 \pm 0.07$ & $37.4 \pm 0.7$ \\
\hline$C_{B u f I b u}$ & Initial buffer isobutyrate, $\mathrm{mmol} / \mathrm{L}$ & $2.02 \pm 0.05$ & $2.08 \pm 0.05$ \\
\hline$k_{\text {PasAq, RuAq }}$ & Ruminal passages rate constant, /h & $0.15 \pm 0.09$ & $0.20 \pm 0.07$ \\
\hline$F L_{\text {RuAq,SalAq }}$ & Saliva flow, L/h & $1.97 \pm 2.43$ & $1.46 \pm 1.46$ \\
\hline$i V_{\mathrm{RuH} 2 \mathrm{O}}$ & Residual ruminal water, $\mathrm{L}$ & $0.95 \pm 0.79$ & $1.47 \pm 0.97$ \\
\hline CPI $(\mathrm{n}=24)$ & $\mathrm{CP}$ intake, $\mathrm{kg} / \mathrm{d}$ & \multicolumn{2}{|c|}{$2.92 \pm 0.57$} \\
\hline $\mathrm{BW}(\mathrm{n}=24)$ & $\mathrm{BW}, \mathrm{kg}$ & \multicolumn{2}{|c|}{$565 \pm 42$} \\
\hline
\end{tabular}

butyrate on epithelial blood flow, VFA fluxes, and VFA concentrations in different pools of the model after a 30min simulation. Sensitivity analysis was also performed on the final model with $1 \%$ variation in the parameter estimates, on the epithelial blood flow, and propionate fluxes between rumen and epithelium. Sensitivity coefficients (response variable/parameter estimates) after a 30-min simulation were obtained from the analysis. The sensitivity analysis on parameters was performed using the algorithm resident in acslX optimizer.

Residual errors of prediction were used to calculate mean square prediction errors (MSPE). The partition of MSPE between mean bias, slope bias, and random dispersion elements was used to evaluate the fit to observed data (Bibby and Toutenberg, 1977). These elements are presented as percentages of MSPE for evaluation purposes. The root mean square prediction error (RMSPE) is presented as a percentage of the observed mean. Model reduction was performed by evaluating the log-likelihood function of the reduced model against the full model using a $\chi^{2}$ test.

\section{RESULTS}

The model was constructed to describe the effects of rumen epithelial blood flow on ruminal absorption of
VFA. The mean inputs to the model are presented in Table 2, and the parameter estimates for rumen epithelial uptake of VFA and rumen epithelial blood flow are presented in Table 3, and the parameter estimates for ruminal uptake of VFA from the epithelial pool of VFA are presented in Table 4.

Epithelial blood flow was calculated from Eq. [13] with consideration of a basal flow affected by CPI per kilogram of BW and a variable flow affected by the butyrate concentration in the ventral rumen. The estimates for $k_{B F C P I}$ and $k_{B F B u}$ were, using Eq. [13], $10.9 \pm$ 0.14 and $0.030 \pm 0.005$, respectively, and they were not correlated $(\mathrm{r}=0.03)$. The epithelial blood flow RMSPE was $26.3 \%$ relative to the observed mean, with no apparent systematic bias when evaluated across all data (Table 5; Figure 2). Root MSPE analysis performed separately for the 2 treatments showed some mean and slope bias for the high butyrate treatment (22.6 and $20.1 \%$ of the MSPE, respectively).

The parameter estimate for the rate constant of epithelial uptake of ruminal butyrate $\left(k_{E p B u, R u B u}\right)$ was 0.97 $\pm 0.02 / \mathrm{h}$ and not correlated to other absorption parameter estimates $(\mathrm{r}<-0.01)$. The RMSPE of ruminal butyrate was $1.40 \mathrm{mmol} / \mathrm{L}$, corresponding to $11.3 \%$ of the observed mean. The predictions had some mean and slope biases when evaluated across all data (17.7

Table 3. Parameter estimates derived from fitting the model to observed data of the washed rumen study (Storm et al., 2011)

\begin{tabular}{|c|c|c|c|c|}
\hline Item & Rate constant description & Estimate & $\mathrm{SD}$ & $\begin{array}{l}\mathrm{SD}, \% \text { of } \\
\text { estimate }\end{array}$ \\
\hline$k_{B F B u}$ & Value for the effect of ruminal butyrate on epithelial blood flow, $\mathrm{L}^{3} /\left(\mathrm{mmol}^{2} \times \mathrm{h}\right)$ & 0.030 & 0.005 & 17 \\
\hline$k_{B F C P I}$ & Value for the effect of $\mathrm{CP}$ intake on epithelial blood flow, $(\mathrm{L} \times \mathrm{d}) / \mathrm{h}$ & 10.9 & 0.14 & 1 \\
\hline$k_{E p A c, R u A c}$ & Epithelia uptake of ruminal acetate, /h & 0.72 & 0.02 & 2 \\
\hline$k_{E p P r, R u P r}$ & Epithelia uptake of ruminal propionate, $/ \mathrm{h}$ & 0.91 & 0.06 & 7 \\
\hline$k_{E p B u, R u B u}$ & Epithelia uptake of ruminal butyrate, /h & 0.97 & 0.02 & 2 \\
\hline$k_{\text {EpIbu,RuIbu }}$ & Epithelia uptake of ruminal isobutyrate, /h & 0.32 & 0.02 & 7 \\
\hline
\end{tabular}


Table 4. Rate constants for the flux of VFA from the epithelium into the rumen calculated from Eq. [20]

\begin{tabular}{llc}
\hline Item & Rate constant description & Estimate \\
\hline$k_{R u A c, E p A c}$ & Epithelia release of acetate to the rumen, /h & 10.6 \\
$k_{R u P r, E p P r}$ & Epithelia release of propionate to the rumen, /h & 13.4 \\
$k_{R u B u, E p B u}$ & Epithelia release of butyrate to the rumen, /h & 14.3 \\
$k_{R u I b u, E p I b u}$ & Epithelia release of isobutyrate to the rumen, /h & 4.78 \\
\hline
\end{tabular}

and $21.4 \%$ of the MSPE, respectively). The mean and slope bias of the prediction of ruminal butyrate are represented graphically in Figure 3 and were more pronounced with the high butyrate treatment than with the control.

Ruminal acetate, propionate, and isobutyrate concentrations were predicted with relatively low RMSPE by the model $(5.86,5.75$, and $4.12 \%$ of observed mean, respectively). The estimates for $k_{E p A c, R u A c}, k_{E p P r, R u P r}$, and $k_{E p I b u, R u I b u}$ were $0.72 \pm 0.02,0.91 \pm 0.06$, and $0.32 \pm$ $0.02 / \mathrm{h}$, respectively, and were not correlated. Ruminal propionate predictions had mean bias, and isobutyrate predictions had slope bias (13.6 and $13.8 \%$ of MSPE, respectively), but they were negligible given the low overall error. Predictions of ruminal acetate concentrations were unbiased (Table 5). These biases are represented graphically in Figures 4, 5, and 6.

Using the derived rate constants (Table 3 and 4) and standard inputs differing only in initial buffer concentrations of butyrate $(4.0$ and $37.4 \mathrm{mmol} / \mathrm{L}$ ) and acetate
(54.9 and $22.9 \mathrm{mmol} / \mathrm{L}$ ), the model predicted epithelial blood flow and fluxes between ruminal fluid, rumen epithelium, and blood (Table 6). The ruminal disappearance of propionate and isobutyrate increased $11 \%$ and $5 \%$ due to the $47 \%$ increase in epithelial blood flow, which was induced by increasing the ruminal butyrate concentration from 4.0 to $37.4 \mathrm{mmol} / \mathrm{L}$. The simulated intraepithelial concentration of propionate and isobutyrate decreased with increasing epithelial blood flow (Table 7). The concentration gradient between rumen fluid and rumen epithelium for propionate was 3.2:1 with low epithelial blood flow, which increased to 4.2:1 when the blood flow increased.

Sensitivity analysis with $1 \%$ variations of the rate constants (Table 3 ) on the response variables $B F$, $F_{E p P r, R u P r}$, and $F_{R u P r, E p P r}$ is presented in Table 8. The sensitivity analysis shows that the effect of varying $k_{B F B u}$ on blood flow and propionate flux between rumen and epithelium increases with increasing ruminal butyrate concentration. The propionate flux between

Table 5. Root mean square prediction errors (RMSPE) and partition of those errors into mean bias, slope bias, and dispersion after fitting the model to observed data

\begin{tabular}{|c|c|c|c|c|c|c|c|}
\hline \multirow[b]{2}{*}{ Item $^{1}$} & \multirow[b]{2}{*}{$\mathrm{n}$} & \multirow[b]{2}{*}{$\begin{array}{c}\text { Observed } \\
\text { mean }\end{array}$} & \multirow[b]{2}{*}{ RMSPE } & \multirow[b]{2}{*}{$\begin{array}{l}\text { RMSPE, \% of } \\
\text { observed mean }\end{array}$} & \multicolumn{3}{|c|}{$\%$ of $\mathrm{MSPE}^{2}$} \\
\hline & & & & & $\begin{array}{l}\text { Mean } \\
\text { bias }\end{array}$ & $\begin{array}{l}\text { Slope } \\
\text { bias }\end{array}$ & Dispersion \\
\hline \multicolumn{8}{|c|}{ High butyrate } \\
\hline$B F$ & 24 & 89.4 & 21.9 & 24.5 & 22.6 & 20.1 & 57.4 \\
\hline$C_{R u A c}$ & 32 & 18.5 & 1.09 & 5.88 & 0.10 & 4.16 & 95.7 \\
\hline$C_{R u P r}$ & 32 & 18.0 & 1.08 & 6.00 & 10.5 & 4.61 & 84.9 \\
\hline$C_{R u B u}$ & 32 & 30.7 & 2.41 & 7.85 & 46.7 & 12.4 & 40.9 \\
\hline$C_{R u I b u}$ & 32 & 1.84 & 0.079 & 4.29 & 9.70 & 28.0 & 62.3 \\
\hline \multicolumn{8}{|l|}{ Control } \\
\hline$B F$ & 48 & 55.0 & 14.7 & 26.8 & 1.06 & 11.4 & 87.6 \\
\hline$C_{R u A c}$ & 64 & 46.1 & 2.53 & 5.50 & 3.03 & 8.10 & 88.9 \\
\hline$C_{R u P r}$ & 64 & 18.4 & 1.04 & 5.63 & 15.4 & 6.45 & 78.1 \\
\hline$C_{R u B u}$ & 64 & 3.28 & 0.17 & 5.26 & 11.3 & 7.92 & 80.8 \\
\hline$C_{R u I b u}$ & 64 & 1.78 & 0.071 & 4.02 & 2.47 & 9.42 & 88.1 \\
\hline \multicolumn{8}{|l|}{ All } \\
\hline$B F$ & 72 & 66.4 & 17.5 & 26.3 & 1.99 & 0.40 & 97.6 \\
\hline$C_{R u A c}$ & 96 & 36.9 & 2.16 & 5.86 & 1.70 & 0.018 & 98.3 \\
\hline$C_{R u P r}$ & 96 & 18.3 & 1.05 & 5.75 & 13.6 & 5.62 & 80.8 \\
\hline$C_{R u B u}$ & 96 & 12.4 & 1.40 & 11.3 & 17.7 & 21.4 & 61.0 \\
\hline$C_{R u I b u}$ & 96 & 1.80 & 0.074 & 4.12 & 0.0089 & 13.8 & 86.2 \\
\hline
\end{tabular}




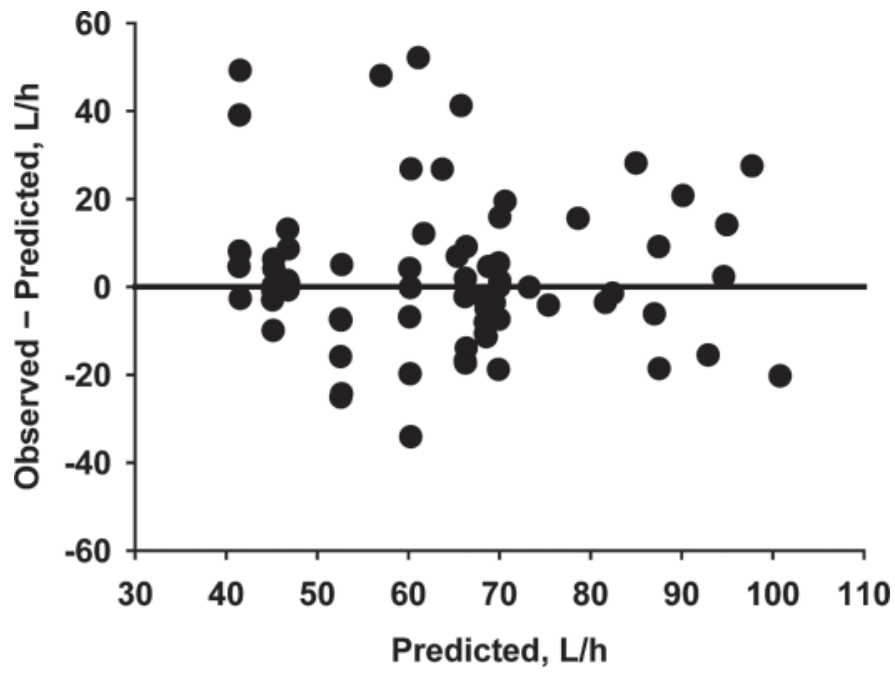

Figure 2. Rumen epithelial blood flow presented as residual versus model prediction. The bold line represents $\mathrm{y}=0$.

rumen and epithelium was highly sensitive to variations in $k_{E p P r, R u P r}$, and the sensitivity seemed to decrease with increasing ruminal concentrations of butyrate.

The model with bidirectional flux and epithelial blood flow was tested against the reduced model without bidirectional flux and epithelial blood flow. The log-likelihood ratio test showed that the more complex model described data better than the reduced model $(P<0.01)$.

\section{DISCUSSION}

Ruminal absorption of VFA can be limited in 3 ways: (1) intraruminal equilibration of VFA between the site of production in the ruminal mat and the site of absorption, (2) permeability of the absorptive epithelium to VFA, or (3) removal of VFA with the blood from the serosal side of the epithelium (Storm and Kristensen, 2010, 2011). Earlier models of VFA absorption from the rumen were derived from ruminal variables, such as chemical composition of the fluid, volume, and $\mathrm{pH}$ (Baldwin et al., 1987; Danfær, 1990; Dijkstra, 1993). Baldwin et al. (1987) related the absorption of ruminal VFA to the ruminal pool size using the same rate constant for all VFA to describe the mass action-driven kinetics. Danfær (1990) used the same technique with different rate constants for each VFA. Dijkstra (1993) was the first to describe ruminal absorption of VFA from a nonlinear approach, using ruminal $\mathrm{pH}$, volume, and chemical composition. Bannink et al. (2008) was the first to incorporate a specific effect of the cow in the control of VFA absorption by using epithelial surface area of the reticulorumen in the model. The present

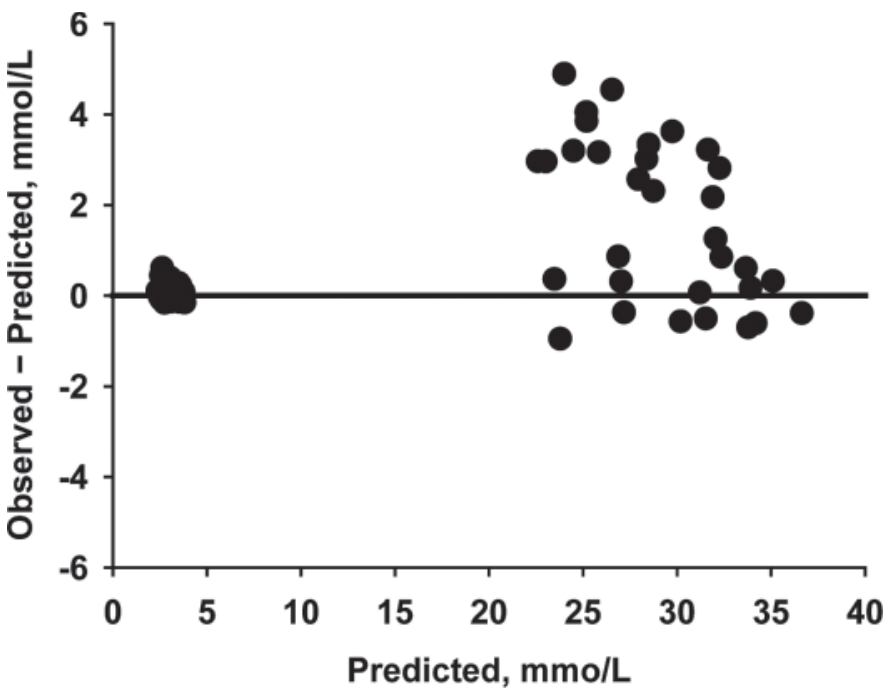

Figure 3. Ruminal butyrate concentrations presented as residual versus model prediction. The bold line represents $\mathrm{y}=0$.

study evaluated the effect of epithelial blood flow on ruminal absorption of VFA (limitation 3) using a modeling approach. The present model was constructed to derive rate constants for the bidirectional flux of VFA between the rumen and epithelium as well as describing the ruminal epithelial blood flow in real time. The model therefore only represents VFA absorption across the ruminal epithelium and does not consider fermentation. Ruminal metabolism is highly complex and is affected by several factors, including diet composition, DMI, stage of lactation, and milk yield. To minimize the effects of these factors on the kinetics data, the

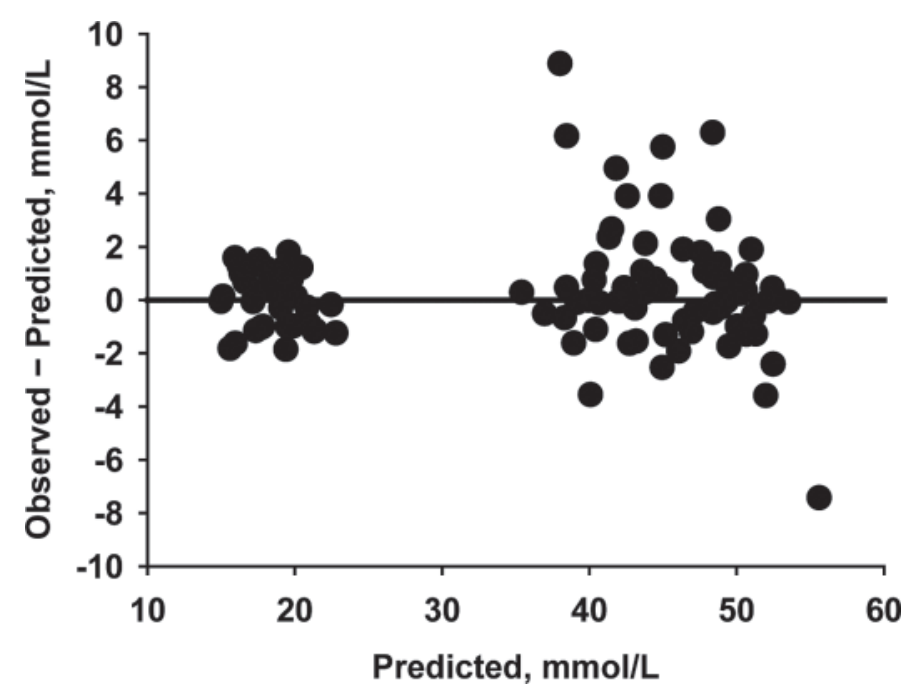

Figure 4. Ruminal acetate concentrations presented as residual versus model prediction. The bold line represents $\mathrm{y}=0$. 


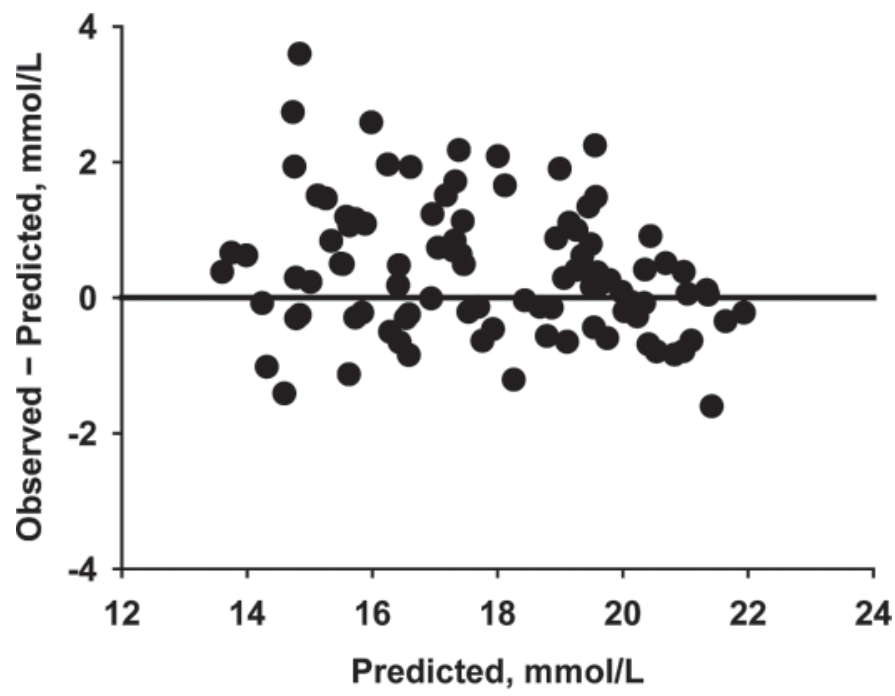

Figure 5. Ruminal propionate concentrations presented as residual versus model prediction. The bold line represents $\mathrm{y}=0$.

washed rumen technique was applied to cows of the same parity (second) and stage of lactation $(63 \pm 4$ DIM). The present model is based on longitudinal data of ruminal VFA concentrations and ruminal epithelial blood flow. The fitted model provided reliable unbiased predictions of ruminal absorption and epithelial blood flow when evaluated against the data of Storm et al. (2011).

\section{General Assumptions}

The ability of a VFA molecule to pass the epithelial membrane was assumed to be the same in both directions if ruminal $\mathrm{pH}$ equals intracellular $\mathrm{pH}$. One way to represent VFA transport across a membrane is

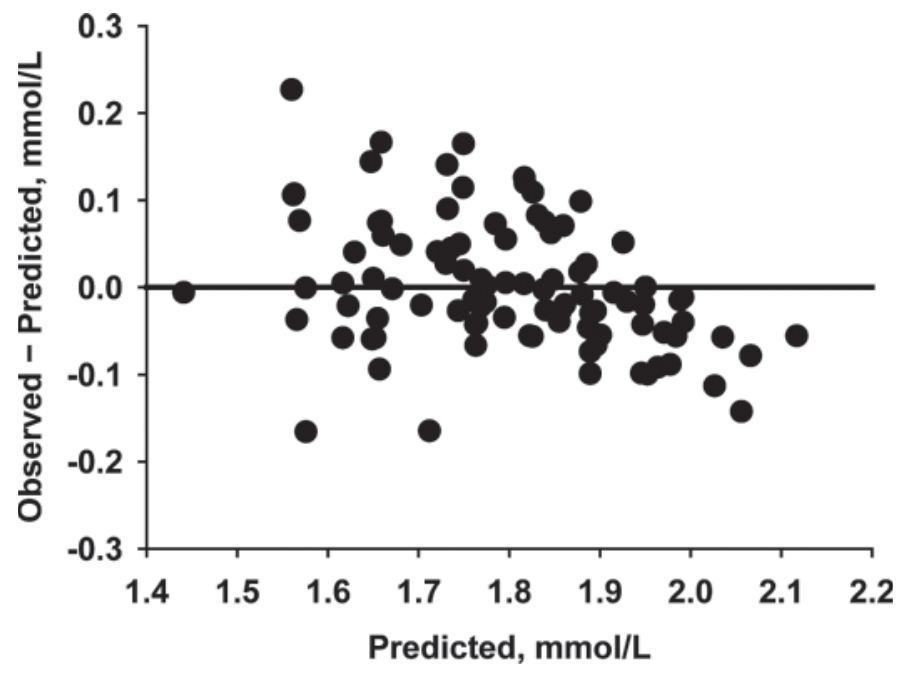

Figure 6. Ruminal isobutyrate concentrations presented as residual versus model prediction. The bold line represents $\mathrm{y}=0$.

by nonionic diffusion (Walter and Gutknecht, 1986), which is the most common description of ruminal VFA absorption. More recently, other routes of absorption have been studied through transport protein mediated absorption (Aschenbach et al., 2011). Monocarboxylate transporters at the basolateral membrane have been identified to mediate the transport of polar substances such as lactate, BHBA, and ionized VFA into the blood (Kirat et al., 2007). Among the studies that suggested protein-dependent VFA absorption were Sehested et al. (1999b) and Aschenbach et al. (2009), who described sodium-dependent and bicarbonate-dependent VFA absorption mechanisms, respectively. Both of these transport proteins are supposedly placed in the apical membrane, but none of these proteins have yet been

Table 6. Mean flux $(\mathrm{mmol} / \mathrm{h})$ predictions ${ }^{1}$ for VFA generated from the model with control or high butyrate input variables, where blood flow predictions were 56.4 and $83.1 \mathrm{~L} / \mathrm{h}$, respectively ${ }^{2}$

\begin{tabular}{|c|c|c|c|c|c|c|c|c|c|}
\hline Item $^{3}$ & Eq. & \multicolumn{2}{|c|}{ Acetate } & \multicolumn{2}{|c|}{ Propionate } & \multicolumn{2}{|c|}{ Butyrate } & \multicolumn{2}{|c|}{ Isobutyrate } \\
\hline$F_{R u V F A, E p V F A}$ & [10] & 293 & 97 & 170 & 127 & 34 & 235 & 2.7 & 2.0 \\
\hline$F_{E p V F A, A b V F A}$ & [11] & 31 & 46 & 1.6 & 2.4 & 2.1 & 3.1 & 0.14 & 0.20 \\
\hline$F_{R V b V F A, E p V F A}$ & {$[12]$} & 746 & 365 & 342 & 377 & 65 & 655 & 15 & 16 \\
\hline
\end{tabular}

${ }^{1}$ The values presented are the mean of 3 simulations using mean inputs and represent the flux from 3 to 9,9 to 20 , and 20 to 30 min as in the washed rumen trial (Storm et al., 2011).

${ }^{2}$ Control: $C_{A b A c}, 0.55 \mathrm{mmol} / \mathrm{L} ; C_{A b P r}, 0.029 \mathrm{mmol} / \mathrm{L} ; C_{A b B u}, 0.0024 \mathrm{mmol} / \mathrm{L} ; C_{A b l b u}, 0.037 \mathrm{mmol} / \mathrm{L} ; C_{B u f A c}, 54.9 \mathrm{mmol} / \mathrm{L} ; C_{B u f P r}, 22.4 \mathrm{mmol} / \mathrm{L}$; $C_{B u f B u}, 4.0 \mathrm{mmol} / \mathrm{L} ; C_{B u f l b u}, 2.08 \mathrm{mmol} / \mathrm{L} ; k_{P a s A q . R u A q}, 0.15 / \mathrm{h}, i V_{R u H 2 O}, 0.95 \mathrm{~L} ; F L_{R u A q . S a l A q}, 1.97 \mathrm{~L} / \mathrm{h} ; \mathrm{CP}$ intake, $2.92 \mathrm{~kg} / \mathrm{d} ; \mathrm{BW}, 565 \mathrm{~kg}$. High butyrate: $C_{A b A c}, 0.55 \mathrm{mmol} / \mathrm{L} ; C_{A b P r}, 0.029 \mathrm{mmol} / \mathrm{L} ; C_{A b B u}, 0.0024 \mathrm{mmol} / \mathrm{L} ; C_{A b I b u}, 0.037 \mathrm{mmol} / \mathrm{L} ; C_{B u f A c}, 22.9 \mathrm{mmol} / \mathrm{L} ; C_{B u f P r}, 22.4 \mathrm{mmol} / \mathrm{L} ; C_{B u f B u}$, $37.4 \mathrm{mmol} / \mathrm{L} ; C_{B u f f b u}, 2.08 \mathrm{mmol} / \mathrm{L} ; k_{\text {PasAq,RuAq }}, 0.15 / \mathrm{h}, i V_{R u H 2 O}, 0.95 \mathrm{~L} ; F L_{R u A q, S a l A q}, 1.97 \mathrm{~L} / \mathrm{h} ; \mathrm{CP}$ intake, $2.92 \mathrm{~kg} / \mathrm{d} ; \mathrm{BW}, 565 \mathrm{~kg}$.

${ }^{3}$ Flux abbreviations are explained in Table 1. 
Table 7. Mean ruminal and epithelial concentrations and the concentration gradient between rumen and epithelia of acetate, propionate, butyrate, and isobutyrate predictions ${ }^{1}$ generated from the model with input data of control and high butyrate ${ }^{2}$

\begin{tabular}{|c|c|c|c|c|c|c|}
\hline \multirow[b]{2}{*}{ Item } & \multicolumn{3}{|c|}{ Control } & \multicolumn{3}{|c|}{ High butyrate } \\
\hline & $\begin{array}{l}\mathrm{C}_{\text {RuVFA }},{ }^{3} \\
\mathrm{mmol} / \mathrm{L}\end{array}$ & $\begin{array}{l}\mathrm{C}_{E p V F A}{ }^{4} \\
\mathrm{mmol} / \mathrm{L}\end{array}$ & Gradient & $\begin{array}{l}\mathrm{C}_{\text {RuVFA }},{ }^{3} \\
\mathrm{mmol} / \mathrm{L}\end{array}$ & $\begin{array}{l}\mathrm{C}_{E P V F A}{ }^{4} \\
\mathrm{mmol} / \mathrm{L}\end{array}$ & Gradient \\
\hline Acetate & 46.1 & 12.6 & $3.7: 1$ & 19.1 & 4.24 & $4.5: 1$ \\
\hline Propionate & 18.3 & 5.74 & $3.2: 1$ & 18.1 & 4.35 & $4.2: 1$ \\
\hline Butyrate & 3.26 & 1.09 & $3.0: 1$ & 29.9 & 7.54 & $4.0: 1$ \\
\hline Isobutyrate & 1.85 & 0.26 & $7.2: 1$ & 1.85 & 0.19 & $9.8: 1$ \\
\hline
\end{tabular}

${ }^{1}$ The values presented are the concentrations of a $30-\min$ simulation using mean inputs.

${ }^{2}$ Control: $C_{A b A c}, 0.55 \mathrm{mmol} / \mathrm{L} ; C_{A b P r}, 0.029 \mathrm{mmol} / \mathrm{L} ; C_{A b B u}, 0.0024 \mathrm{mmol} / \mathrm{L} ; C_{A b I b u}, 0.037 \mathrm{mmol} / \mathrm{L} ; C_{B u f A c}, 54.9$ $\mathrm{mmol} / \mathrm{L} ; C_{B u f P r}, 22.4 \mathrm{mmol} / \mathrm{L} ; C_{B u f B u}, 4.0 \mathrm{mmol} / \mathrm{L} ; C_{B u f I b u}, 2.08 \mathrm{mmol} / \mathrm{L} ; k_{P a s A q, R u A q}, 0.15 / \mathrm{h} ; i V_{R u H 2 O}, 0.95 \mathrm{~L}$; $F L_{R u A q, S a l A q}, 1.97 \mathrm{~L} / \mathrm{h} ; \mathrm{CP}$ intake, $2.92 \mathrm{~kg} / \mathrm{d} ; \mathrm{BW}, 565 \mathrm{~kg}$. High butyrate: $C_{A b A c}, 0.55 \mathrm{mmol} / \mathrm{L} ; C_{A b P r}, 0.029$ $\mathrm{mmol} / \mathrm{L} ; C_{A b B u}, 0.0024 \mathrm{mmol} / \mathrm{L} ; C_{A b l b u}, 0.037 \mathrm{mmol} / \mathrm{L} ; C_{B u f A c}, 22.9 \mathrm{mmol} / \mathrm{L} ; C_{B u f P r}, 22.4 \mathrm{mmol} / \mathrm{L} ; C_{B u f B u}, 37.4$ $\mathrm{mmol} / \mathrm{L} ; C_{\text {Bufflbu}}, 2.08 \mathrm{mmol} / \mathrm{L} ; k_{\text {PasAq, RuAq }}, 0.15 / \mathrm{h} ; i V_{\text {RuH2O }}, 0.95 \mathrm{~L} ; F L_{\text {RuAq,SalAq }}, 1.97 \mathrm{~L} / \mathrm{h} ;$ CP intake, $2.92 \mathrm{~kg} / \mathrm{d}$; $\mathrm{BW}, 565 \mathrm{~kg}$.

${ }^{3}$ Mean ruminal concentration of VFA, Eq. [4].

${ }^{4}$ Mean epithelial concentration of VFA, Eq. [5].

identified (Aschenbach et al., 2011). In a recent review by Aschenbach et al. (2011), it was estimated that ruminal absorption of VFA through protein-mediated transport might account for as much as 50\%. The kinetics of protein-mediated transport suggests a saturable process, best modeled through a Michaelis-Menten equation to access the full range of substrate concentrations. Within the linear range of a saturable process, the kinetics can be described by mass action, which is the most common representation of ruminal VFA absorption. In the present work, the model does not depend on the mechanism by which VFA is transported as long as the transport is driven by mass action in the range of ruminal VFA concentrations used. Considering the residual plots (Figures 4 to 6) and RMSPE (Table 5), model errors appear to be linear, supporting the mass action representation of ruminal acetate, propionate, and isobutyrate absorption. The level of ruminal butyrate used in the high butyrate treatment was initially above common levels and may have changed the conditions of ruminal butyrate absorption. The residual plot (Figure 3) and the MSPE analysis (Table 5) indicated some mean bias for ruminal butyrate with the high butyrate treatment. The ruminal butyrate RMSPE was 5.26 and $7.85 \%$ of the observation mean for control and high butyrate treatments, respectively, and $11.3 \%$ when evaluated across all data. The mean bias of ruminal butyrate indicated that the kinetics of ruminal butyrate absorption is not the same for high and low ruminal concentrations. However, Dijkstra et al. (1993) were not able to find an effect on the fractional absorption rate of butyrate when ruminal butyrate concentration was increased from 20 to $100 \mathrm{mmol} / \mathrm{L}$, concentrations more than twice that used in Storm et al. (2011). Dijkstra et al. (1993) observed an effect of increasing ruminal propionate and acetate concentrations on the fractional absorption rate of these VFA. However, data on the fractional absorption rate of propionate and acetate from that study were confounded with butyrate and were difficult to interpret in the light of the epithelial blood flow effect of butyrate described by Storm et al. (2011).

The concentration of nonionized VFA depends on $\mathrm{pH}$, as described by the Henderson-Hasselbalch equation. With nonionic diffusion, a $\mathrm{pH}$ difference across the membrane can drive VFA transport, even with the same overall concentration of VFA on both sides. Thus, $\mathrm{pH}$ equilibrium between rumen and epithelium was important for model construction. The observed ruminal $\mathrm{pH}$ was, on average, $7.3 \pm 0.13$ and equal to the blood $\mathrm{pH}$ of the right ruminal vein $(7.3 \pm 0.05)$. We therefore assumed no $\mathrm{pH}$ difference between rumen fluid, rumen epithelium, and rumen epithelial blood. Ruminal $\mathrm{pH}$ near neutral is only found in ruminants deprived of feed, and the level used in the present study was therefore not physiologically valid. Ruminal $\mathrm{pH}$ is known to affect the ruminal absorption rate of propionate and butyrate (Dijkstra et al., 1993), and ruminal pH may therefore be an important factor when modeling ruminal VFA absorption. Unfortunately, our data do not support a representation that includes the level of VFA ionization, because epithelial $\mathrm{pH}$ was not obtained. Equal ruminal fluid and blood $\mathrm{pH}$ enabled us to estimate the rate constants for the bidirectional fluxes of VFA between ruminal fluid and ruminal epithelium. The rate constants $\left(k_{R u V F A, E p V F A}\right.$ and $\left.k_{E p V F A, R u V F A}\right)$ were considered proportional to the relative pool size $Q_{R u}$ ${ }_{V F A} / Q_{E P V A A}$ derived from the theoretical situation when 
epithelial blood flow equals zero. Under this condition, the bidirectional fluxes are equal and can be calculated from Eq. [20]. Thus, only one rate constant needs to be estimated because the other can be calculated. Ruminal volume was given on buffer entry, but ruminal epithelial volume was obtained from extrapolating the combined epithelial volume of reticulum, atrium, and ventral ruminal sac of sheep (Barnes et al., 1983) to the observed mean BW of the cows. The epithelial volume was considered constant and not related to the type of diet, even though diet has been observed to have long-term effects on papillation of the rumen (Sakata and Tamate, 1978, 1979). Effect of diet on blood flow was accounted for through the CPI constant $k_{B F C P I}$ in Eq. [13]. However, long-term effects of diet (more than 3 wk) may alter the proportion of blood draining the ruminal epithelium.

Saliva secretion in dairy cows is approximately 240 $\mathrm{L} / \mathrm{d}$, with daily fluctuations affected by rumination time and feed dry matter (Cassida and Stokes, 1986; Maekawa et al., 2002). Saliva flow to the rumen dilutes the ruminal fluid and reduces the concentration difference between rumen and epithelium, and may thereby decrease the rate of ruminal absorption. Ruminal influx of fluid across the rumen wall may also dilute the rumen pool of VFA. Osmolarity of the ruminal fluid is the main determinant of ruminal influx of water across the rumen wall (Warner and Stacy, 1972). To reduce the influx of fluid, isotonic buffer solutions were used. However, the experimental setup was not tuned to distinguish between saliva and fluid influx across the rumen wall or to measure the influx in real time. Saliva flow was therefore a measure of the overall influx of fluid to the rumen and assumed to be constant. Ruminal influx of saliva was assumed to have VFA concentrations equal to those of arterial blood (Eq. [6]). The highest rate of salivation was $8.1 \mathrm{~L} / \mathrm{h}$, providing the rumen with approximately $5 \mathrm{mmol}$ of acetate per hour. Dilution of ruminal fluid with saliva is therefore by far the most important effect of saliva on the absorption under washed rumen conditions.

The passage of liquid and associated VFA out of the rumen was considered driven by mass action. This assumption is not valid for short-term trials under normal ruminal conditions, because passage is related to the ruminal contractions and ruminal fill (Sissons et al., 1984). There are no mechanical stimuli of ruminal contractions under washed rumen conditions, and the frequency of contractions is therefore assumed negligible. The mixing of ruminal fluid was, for the same reason, maintained by agitation of the buffer solutions with a gas mixture. The assumption of ruminal passage as driven by mass action is therefore in agreement with the limitations of the experimental setup. 


\section{Epithelial Blood Flow}

Several studies have shown an effect of ruminal butyrate on ruminal blood supply and ruminal epithelial blood flow (Dobson et al., 1971; Rémond et al., 1993). The site of the responsible receptor for vasodilation has not been located and may be activated by substrate other than butyrate (e.g., BHBA). For modeling purposes, the ruminal compartment was chosen as the location of activation and ruminal butyrate concentration as the controlling factor, in agreement with rumen epithelial chemoreceptors being stimulated by ruminal infusion of butyrate (Upton et al., 1976).

The epithelial blood flow of the active absorptive epithelium was measured as clearance of deuterium oxide $\left(\mathrm{D}_{2} \mathrm{O}\right)$ from the rumen. This method appears relatively robust but has not been evaluated against other methods, and systematic underprediction cannot be excluded. Cow-specific parameters such as epithelial mass, prior incidence of ruminal acidosis, or variation in rumen epithelial capacity between cows might be responsible for variation in data not accounted for by the effect of ruminal butyrate and $\mathrm{CPI} / \mathrm{kg}$ of $\mathrm{BW}$ on epithelial blood flow (RMSPE $26 \%$ of observed mean). Carbon dioxide has previously been observed to affect epithelial blood flow when infused into the rumen (Dobson et al., 1971). Other unknown ruminal and epithelial components, as well as nervous stimuli, may therefore also affect epithelial blood flow. The quadratic effect of ruminal butyrate concentration was evaluated to give the best fit to observed data compared with a linear effect. Physiologically epithelial blood flow is more likely to follow saturation kinetics, with an upper capacity of blood flow related to the size of the cow. The prediction of epithelial blood flow by the model is therefore only valid for ruminal butyrate concentrations $<37.4$ $\mathrm{mmol} / \mathrm{L}$, which was the uppermost concentration in the trial; blood flow estimated for butyrate concentrations above this value may be overestimated. The use of a quadratic equation when estimating blood flow is justified from the span of measurements of epithelial blood flow obtained with ruminal butyrate from 2.50 to 3.64 $\mathrm{mmol} / \mathrm{L}$ and from 22.9 to $33.6 \mathrm{mmol} / \mathrm{L}$. The sensitivity analysis of the 2 parameters affecting epithelial blood flow shows that blood flow is not as sensitive to changes in $k_{B F C P I}$ as it is to changes in $k_{B F B u}$. The sensitivity analysis also shows that epithelial blood flow and propionate fluxes between rumen and epithelium become more sensitive to changes in $k_{B F B u}$ as ruminal butyrate concentrations increase, indicating the dual effect of blood flow and permeability of the epithelium to VFA on ruminal absorption of VFA.

A numerical reduction in blood flow was observed with high ammonia concentrations in the washed rumen study for cows on high CP diets. Initially, a ruminal ammonia pool and urea recycling were included in the model to assess the proposed negative effect of ruminal ammonia on epithelial blood flow. Ruminal ammonia is linked to urea through urea recycling and microbial urease activity associated with rumen contents and ruminal epithelium (Wallace et al., 1979). Ruminal recycling of urea was assumed to be inversely proportional to the CPI, and the ruminal hydrolysis of urea into ammonia, as well as the bidirectional flux between rumen and epithelia of ammonia, was considered to be driven by mass action. The ruminal concentration of ammonia was predicted with $18 \%$ RMSPE of observation mean and with a mean bias of $30 \%$, indicating that some variance was not captured with this interpretation. The poor equilibration between epithelial and ruminal ammonia (Kristensen et al., 2010) might add to the bias on ruminal ammonia concentrations. The bias on ruminal ammonia concentration could also be related to urea recycling across the rumen epithelium because ruminal urea concentration was poorly predicted (44\% RMSPE of observation mean). The initial concentration of urea in the ruminal buffers used experimentally was above common physiological levels and resulted in a positive net portal flux of urea in the first minutes after ruminal introduction. Therefore, the representation of ruminal ammonia and urea was excluded from the model because it was not supported by data.

Using Eq. [13], the model was able to simulate increasing blood flow in the epithelium with increasing butyrate concentrations in the rumen. Under standard conditions with manipulation of ruminal butyrate (4.0 to $37.4 \mathrm{mmol} / \mathrm{L}$ ) and acetate (54.9 to $22.9 \mathrm{mmol} / \mathrm{L})$, epithelial blood flow increased $47 \%$ with high butyrate compared with control simulations. For the same manipulation of ruminal acetate and butyrate, the observed epithelial blood flow increased $49 \%$ (Storm et al., 2011). The residual variation to predicted epithelial blood flow was high and presumably related to difference between cows not captured by the model.

\section{Ruminal Absorption of VFA}

All VFA fluxes between rumen and epithelium in the present model were considered driven by mass action. Thus, intraepithelial concentrations of VFA were linked to removal of VFA by blood. With decreasing blood flow, intraepithelial concentrations would increase, resulting in increased flux of VFA from the epithelium to the rumen. The bidirectional fluxes are therefore of great importance when modeling VFA absorption with low epithelial blood flow. Bidirectional absorption of acetate and propionate has been observed across ruminal epithelium in vitro (Stevens and Stettler, 
1967; Sehested et al., 1999a) and for acetate in vivo (Kristensen et al., 1996). Ruminal acetate, propionate, and isobutyrate were all well represented in the model with low MSPE (RMSPE $\leq 7.85 \%$ of observation mean: Table 5) and linear residuals (Figures 4 to 6), supporting the concept of describing transport of these VFA by mass action and epithelial blood flow.

With concentration gradient-driven absorption, extensive epithelial metabolism can increase the absorption rate by reducing cellular concentrations of VFA. We assumed no epithelial metabolism of any VFA, which is not in agreement with the general understanding of rumen epithelial function. Butyrate is extensively metabolized into BHBA and acetoacetate (Weigand et al., 1972; Sehested et al., 1999a; Kristensen and Harmon, 2004a). The metabolic capacity of the ruminal epithelium to butyrate might be related to the physiological conditions of the rumen, and long-term adaptation to higher levels of butyrate (VFA) may increase the capacity (Sehested et al., 2000). Kristensen and Harmon (2004a) evaluated the effect of a short-term increase of ruminal butyrate concentrations in steers on the recovery of butyrate in portal blood. Kristensen and Harmon (2004a) found the epithelial capacity to metabolize butyrate to be limited, in line with the metabolism being under enzymatic control. The intraepithelial concentration of butyrate is therefore affected by the rate of conversion, and the intraepithelial concentrations of butyrate presented in Table 7 are therefore not biologically valid.

Initially, the present model was fitted with a Michaelis-Menten equation to represent the epithelial metabolism of butyrate into BHBA, using the epithelial pool of butyrate as substrate. Longitudinal data on net ruminal vein flux of butyrate and BHBA were used with the optimizer to fit an affinity constant $\left(\boldsymbol{k}_{\boldsymbol{m}}\right)$ and the maximal process velocity $\left(\boldsymbol{V}_{\max }\right)$ of the enzymatic process. A direct estimation of $k_{m}$ and $V_{\max }$ of this process was not possible using these data. Unfortunately, data on net ruminal vein flux were not well characterized for cows on low $\mathrm{CP}$ because of missing ruminal vein samples from 2 cows in the washed rumen study, which might have corrupted the direct estimation of $k_{m}$ and $V_{\max }$. An attempt to estimate $V_{\max }$ by setting $k_{m}$ to a literature value of butyryl-CoA synthetase (EC 6.2.1.2: $k_{m}$ $=6.8$; Scaife and Tichivangana, 1980) did not result in improved representation of the ruminal concentration of butyrate. Similarly, the attempt to estimate $k_{m}$ by setting $V_{\max }$ to the mean net portal flux of BHBA ( $V_{\max }$ $=192 \mathrm{mmol} / \mathrm{h}$ ) observed in the washed rumen study with the high butyrate buffer was not successful (Storm et al., 2011). The literature affinity coefficient $\left(k_{m}=\right.$ 6.8) was chosen because it was estimated from ovine ruminal epithelium (Scaife and Tichivangana, 1980).
However, the substrate concentration is important for representing the epithelial metabolism and, as the epithelial pool of butyrate is likely to be overestimated by the model, this together with other errors may have corrupted the estimation of $V_{\max }$. Although the constant $V_{\max }$ used $(192 \mathrm{mmol} / \mathrm{h})$ was obtained under experimental conditions with a relatively high epithelial conversion of butyrate (Storm et al., 2011), $V_{\max }$ may not have been reached under these conditions. Reliable estimates of $k_{m}$ and $V_{\max }$ may not be obtained from data of net ruminal vein fluxes of butyrate and BHBA because epithelial conversion of butyrate is presumably regulated by more than one enzyme and the products are compounds other than BHBA (e.g., acetoacetate).

Propionate is another VFA extensively metabolized by splanchnic tissues, primarily the liver, but approximately $13 \%$ of ruminal-absorbed propionate is metabolized in the epithelium of cattle (Kristensen and Harmon, 2004a). The main product of epithelial propionate is expected to be L-lactate. The model was therefore initially constructed with an L-lactate pool in the epithelium and the conversion of epithelial propionate to L-lactate through a flat rate transformation $(13 \%)$. We did not consider fitting the metabolism of propionate to the ruminal vein flux of L-lactate for the same reasons as for butyrate, together with the observation that Llactate is the oxidation product of other pathways, especially valerate $\beta$-oxidation (Kristensen and Harmon, 2005). In the final model, no metabolism of propionate was applied because it did not improve the fit of ruminal propionate. Acetate and isobutyrate were not considered to be metabolized in the epithelium because the observed recovery into the ruminal vein blood was 110 and $93 \%$ in the washed rumen study, respectively, which is in agreement with other studies (Kristensen and Harmon, 2004a,b). Acetate was the ruminal VFA with the lowest residual error, underlining the effect of bidirectional flux and epithelial blood flow on ruminal VFA kinetics under the experimental conditions.

The rate constants for ruminal VFA absorption were in the order isobutyrate $<$ acetate $<$ propionate $<$ butyrate, which is in agreement with several studies in cattle under intact (Krehbiel et al., 1995) and washed (Dijkstra et al., 1993; Kristensen and Harmon, 2004a,b) rumen conditions. In these studies, ruminal absorption is presented as a unidirectional flux and the rate constants are therefore lower, in general, than in the present model.

As in the washed rumen study, we used propionate and isobutyrate as VFA markers in the simulation because acetate and butyrate were manipulated across simulations. The observed effect of epithelial blood flow on net portal flux of propionate and isobutyrate was 31 and 10\%, respectively, with a $49 \%$ change in 
epithelial blood flow (Storm et al., 2011). However, the net portal flux is a cumulative flux accounting for hindgut production, passages to the omasum, as well as portal-drained viscera uptake of VFA (metabolism and catabolism). The increase in net ruminal vein flux in the present model was not as great as the observed changes in net portal flux but was in the same order $(10$ and 5\% for propionate and isobutyrate, respectively). Because of the anatomical difference between net portal flux and net ruminal vein flux, a direct comparison was not possible. The ruminal disappearance of propionate predicted was $11 \%$, only 2 percentage units less than the observed value (Storm et al., 2011). This indicates that the model was in agreement with the observed disappearance of propionate. However, the variation in blood response suggested that epithelial metabolism was an important determinant of ruminal and portal vein flux of VFA.

The simulation indicated that the rumen to intraepithelial concentration gradient of propionate was no more than 3:1 and increasing with blood flow to 4:1, signifying that the permeability of the ruminal epithelium becomes increasingly important in control of VFA flux with increasing epithelial blood flow. The extent of epithelial metabolism may affect this concentration gradient, as mentioned earlier. Ruminal fluid to epithelial blood gradients of propionate were approximately 5:1 in the washed rumen study. With $13 \%$ epithelial metabolism of propionate, the model-predicted gradient would increase. The concentration gradient is inversely proportional to the absorption rate constant (Table 7); that is, butyrate has the lowest and isobutyrate the highest concentration gradient. This demonstrates that the response of varying epithelial blood flow depends on the permeability of the compound absorbed, which is supported by the lower incremental increase of net portal flux of isobutyrate compared with propionate (Storm et al., 2011). A change in ruminal $\mathrm{pH}$ affects VFA dissociation, but the intraepithelial concentration may not follow ruminal $\mathrm{pH}$, and the effective concentration gradient of nonionized VFA is not known. This aspect of the study needs more attention experimentally.

\section{Perspectives}

As stated earlier, the VFA absorption from the rumen can be limited in 3 ways: (1) intraruminal equilibration of VFA between the site of production in the ruminal mat and the site of absorption, (2) permeability of the absorptive epithelium to VFA, or (3) removal of VFA with the blood from the serosal side of the epithelium. Future feed evaluation systems based on dynamic simulation models have to consider all 3 components.
The data used for fitting the model were obtained under washed rumen conditions and the model was constructed around this scenario; therefore, in its current form it is not applicable for more physiologically correct models. The model requires several inputs as arterial and ruminal concentrations of VFA and does not consider ruminal VFA production; however, subelements of the model may be beneficial for existing models. The current study clearly supports the importance of implementing epithelial blood flow and bidirectional fluxes in existing VFA models based on mass action and unidirectional absorption, such as Baldwin et al. (1987) and Danfær (1990). The introduction of Eq. [13] in existing mechanistic VFA absorption models is only relevant if bidirectional flux is also applied. The present model may be incorporated as an absorption model from the liquid phase of the ventral rumen, assuming this site to be the main site of VFA absorption. The medial mat of structural material has been shown to be the primary site of fermentation, and the turnover of VFA from the mat is relatively lower than the absorption rate from the ventral rumen (Storm and Kristensen, 2010, 2011). Because of the lower turnover of VFA in the mat, ruminal fermentation is controlled by chemical parameters of the mat rather than by chemical properties of the ventral rumen. Separating ruminal models into fermentation and absorption submodels may therefore give a more accurate estimation of the complexity of the rumen. However, in the prediction of true net ruminal vein flux of VFA, the present model lacks epithelial metabolism of propionate and butyrate as well as the effect of ruminal and intercellular $\mathrm{pH}$ on ruminal absorption. These effects are undoubtedly important when modeling the net ruminal vein fluxes of VFA within the common range of ruminal $\mathrm{pH}$.

\section{CONCLUSIONS}

A dynamic model based on rumen epithelial blood flow and bidirectional flux of VFA between ruminal fluid and epithelium gave unbiased predictions with low residual error of ruminal absorption of VFA under washed rumen conditions. The control of ruminal VFA absorption in relation to varying epithelial blood flow was related to the concentration gradient between ruminal fluid and epithelial blood. This emphasizes the importance of including properties of rumen epithelial membranes, epithelial metabolism, and epithelial blood flow in future models of ruminal VFA kinetics and ruminal environment. However, these conclusions have not been tested on independent data and further research is therefore needed. 


\section{ACKNOWLEDGMENTS}

We gratefully acknowledge Birgit H. Løth, Anne Krustrup, Mogens Larsen, Pia Jensen, Marie Engbæk, Thorkild N. Jakobsen, Ole H. Olsen, and the barn staff at Faculty of Agricultural Sciences, Aarhus University (Tjele, Denmark), as well as Robin McDougall from The AEgis Technologies Group Inc. (Huntsville, AL) for skillful technical and expert assistance. This project was funded by the Commission of the European Communities (Brussels, Belgium; FP7, KBBE-2007-1), the Directorate for Food, Fisheries and Agri Business (Copenhagen, Denmark; \#3304-VMP-05-005), the Danish Cattle Federation (\#Stivelsesprojektet; Aarhus, Denmark), and the Danish Ministry of Food, Agriculture and Fisheries (Copenhagen, Denmark).

\section{REFERENCES}

Aschenbach, J. R., S. Bilk, G. Tadesse, F. Stumpff, and G. Gabel. 2009. Bicarbonate-dependent and bicarbonate-independent mechanisms contribute to nondiffusive uptake of acetate in the ruminal epithelium of sheep. Am. J. Physiol. Gastrointest. Liver Physiol. 296:G1098-G1107.

Aschenbach, J. R., G. B. Penner, F. Stumpff, and G. Gäbel. 2011. Ruminant nutrition symposium: Role of fermentation acid absorption in the regulation of ruminal pH. J. Anim. Sci. 89:1092-1107.

Baldwin, R. L., J. H. M. Thornley, and D. E. Beever. 1987. Metabolism of the lactating cow. 2. Digestive elements of a mechanistic model. J. Dairy Res. 54:107-131.

Bannink, A., H. De Visser, A. Klop, J. Dijkstra, and J. France. 1997a. Causes of inaccurate prediction of volatile fatty acids by simulation models of rumen function in lactating cows. J. Theor. Biol. 189:353-366

Bannink, A., H. De Visser, and A. M. Van Vuuren. 1997b. Comparison and evaluation of mechanistic rumen models. Br. J. Nutr. 78:563-581.

Bannink, A., J. France, S. Lopez, W. J. J. Gerrits, E. Kebreab, S. Tamminga, and J. Dijkstra. 2008. Modelling the implications of feeding strategy on rumen fermentation and functioning of the rumen wall. Anim. Feed Sci. Technol. 143:3-26.

Bannink, A., J. Kogut, J. Dijkstra, J. France, E. Kebreab, A. M. Van Vuuren, and S. Tamminga. 2006. Estimation of the stoichiometry of volatile fatty acid production in the rumen of lactating cows. J. Theor. Biol. 238:36-51.

Barnes, R. J., R. S. Comline, and A. Dobson. 1983. Changes in the blood flow to the digestive organs of sheep induced by feeding. Q. J. Exp. Physiol. 68:77-88.

Bergman, E. N. 1990. Energy contributions of volatile fatty-acids from the gastrointestinal-tract in various species. Physiol. Rev. 70:567-590.

Bibby, J., and H. Toutenberg. 1977. Prediction and Improved Estimation in Linear Models. Wiley, Chichester, UK.

Bugaut, M. 1987. Occurrence, absorption and metabolism of short chain fatty acids in the digestive tract of mammals. Comp. Biochem. Physiol. B 86:439-472.

Cassida, K. A., and M. R. Stokes. 1986. Eating and resting salivation in early lactation dairy cows. J. Dairy Sci. 69:1282-1292.

Danfær, A. 1990. A dynamic model of nutrient digestion and metabolism in lactating dairy cows. Thesis. Report 671, National Institute of Animal Science, Foulum, Denmark.

Dijkstra, J. 1993. Mathematical modeling and integration of rumen fermentation processes. PhD Thesis. Agricultural University of Wageningen, Wageningen, the Netherlands.
Dijkstra, J. 1994. Production and absorption of volatile fatty acids in the rumen. Livest. Prod. Sci. 39:61-69.

Dijkstra, J., H. Boer, J. Vanbruchem, M. Bruining, and S. Tamminga. 1993. Absorption of volatile fatty acids from the rumen of lactating dairy-cows as influenced by volatile fatty acid concentration, ph and rumen liquid volume. Br. J. Nutr. 69:385-396.

Dobson, A., A. F. Sellers, and S. O. Thorlaci. 1971. Limitation of diffusion by blood flow through bovine ruminal epithelium. Am. J. Physiol. 220:1337-1343.

Kirat, D., Y. Matsuda, N. Yamashiki, H. Hayashi, and S. Kato. 2007. Expression, cellular localization, and functional role of monocarboxylate transporter 4 (MCT4) in the gastrointestinal tract of ruminants. Gene 391:140-149.

Krehbiel, C. R., K. R. A. Britton, D. L. Harmon, T. J. Wester, and R. A. Stock. 1995. The effects of ruminal acidosis on volatile fatty acid absorption and plasma activities of pancreatic enzymes in lambs. J. Anim. Sci. 73:3111-3121.

Kristensen, N. B., A. Danfær, V. Tetens, and N. Agergaard. 1996. Portal recovery of intraruminally infused short-chain fatty acids in sheep. Acta Agric. Scand. A 46:26-38.

Kristensen, N. B., and D. L. Harmon. 2004a. Effect of increasing ruminal butyrate absorption on splanchnic metabolism of volatile fatty acids absorbed from the washed reticulorumen of steers. J. Anim. Sci. 82:3549-3559.

Kristensen, N. B., and D. L. Harmon. 2004b. Splanchnic metabolism of volatile fatty acids absorbed from the washed reticulorumen of steers. J. Anim. Sci. 82:2033-2042.

Kristensen, N. B., and D. L. Harmon. 2005. Effects of adding valerate, caproate, and heptanoate to ruminal buffers on splanchnic metabolism in steers under washed-rumen conditions. J. Anim. Sci. 83:1899-1907.

Kristensen, N. B., A. C. Storm, and M. Larsen. 2010. Effect of dietary nitrogen content and intravenous urea infusion on ruminal and portal-drained visceral extraction of arterial urea in lactating Holstein cows. J. Dairy Sci. 93:2670-2683.

Maekawa, M., K. A. Beauchemin, and D. A. Christensen. 2002. Effect of concentrate level and feeding management on chewing activities, saliva production, and ruminal $\mathrm{pH}$ of lactating dairy cows. J. Dairy Sci. 85:1165-1175.

Morvay, Y., A. Bannink, J. France, E. Kebreab, and J. Dijkstra. 2011. Evaluation of models to predict the stoichiometry of volatile fatty acid profiles in rumen fluid of lactating Holstein cows. J. Dairy Sci. 94:3063-3080.

Murphy, M. R., R. L. Baldwin, and L. J. Koong. 1982. Estimation of stoichiometric parameters for rumen fermentation of roughage and concentrate diets. J. Anim. Sci. 55:411-421.

Rémond, D., J. P. Chaise, E. Delval, and C. Poncet. 1993. Net transfer of urea and ammonia across the ruminal wall of sheep. J. Anim. Sci. $71: 2785-2792$.

Reynolds, C. K., and G. B. Huntington. 1988. Partition of portaldrained visceral net flux in beef steers. Br. J. Nutr. 60:553-562.

Sakata, T., and H. Tamate. 1978. Rumen epithelial cell proliferation accelerated by rapid increase in intraruminal butyrate. J. Dairy Sci. 61:1109-1113.

Sakata, T., and H. Tamate. 1979. Rumen epithelium cell proliferation accelerated by propionate and acetate. J. Dairy Sci. 62:49-52.

Scaife, J. R., and J. Z. Tichivangana. 1980. Short chain acyl-CoA synthetases in ovine rumen epithelium. Biochim. Biophys. Acta 619:445-450.

Sehested, J., J. B. Andersen, O. Aaes, N. B. Kristensen, L. Diernæs, P. D. Møller, and E. Skadhauge. 2000. Feed-induced changes in the transport of butyrate, sodium and chloride ions across the isolated bovine rumen epithelium. Acta Agric. Scand. A 50:47-55.

Sehested, J., L. Diernæs, P. D. Møller, and E. Skadhauge. 1999a. Ruminal transport and metabolism of short-chain fatty acids (SCFA) in vitro: Effect of SCFA chain length and $\mathrm{pH}$. Comp. Biochem. Physiol. A Mol. Integr. Physiol. 123:359-368.

Sehested, J., L. Diernæs, P. D. Møller, and E. Skadhauge. 1999b. Transport of butyrate across the isolated bovine rumen epithelium: Interaction with sodium, chloride and bicarbonate. Comp. Biochem. Physiol. A Mol. Integr. Physiol. 123:399-408. 
Sissons, J. W., S. M. Thurston, and R. H. Smith. 1984. Reticular myoelectric activity and turnover of rumen digesta in the growing steer. Can. J. Anim. Sci. 64:70-71.

Stevens, C. E., and B. K. Stettler. 1967. Evidence for active transport of acetate across bovine rumen epithelium. Am. J. Physiol. 213:1335-1339.

Storm, A. C., M. D. Hanigan, and N. B. Kristensen. 2011. Effects of ruminal ammonia and butyrate concentrations on reticuloruminal epithelial blood flow and VFA absorption kinetics under washed reticulorumen conditions in lactating dairy cows. J. Dairy Sci. 94:3980-3994.

Storm, A. C., and N. B. Kristensen. 2010. Effects of particle size and dry matter content of a total mixed ration on intraruminal equilibration and net portal flux of volatile fatty acids in lactating dairy cows. J. Dairy Sci. 93:4223-4238.

Storm, A. C., and N. B. Kristensen. 2011. Erratum to "Effects of particle size and dry matter content of a total mixed ration on in- traruminal equilibration and net portal flux of volatile fatty acids in lactating dairy cows" (J. Dairy Sci. 93:4223-4238). J. Dairy Sci. 94:532-535.

Upton, P. K., J. P. Ryan, and B. F. Leek. 1976. Acid as a sensory stimulus in the alimentary canal (sheep). Ir. J. Med. Sci. 145:307-308.

Wallace, R. J., K. J. Cheng, D. Dinsdale, and E. R. Ørskov. 1979. An independent microbial flora of the epithelium and its role in the ecomicrobiology of the rumen. Nature 279:424-426.

Walter, A., and J. Gutknecht. 1986. Permeability of small nonelectrolytes through lipid bilayer membranes. J. Membr. Biol. 90:207217.

Warner, A. C., and B. D. Stacy. 1972. Water, sodium and potassium movements across the rumen wall of sheep. Q. J. Exp. Physiol Cogn. Med. Sci. 57:103-119.

Weigand, E., J. W. Young, and A. D. McGilliard. 1972. Extent of butyrate metabolism by bovine ruminoreticulum epithelium and the relationship to absorption rate. J. Dairy Sci. 55:589-597. 